OPEN ACCESS

Edited by:

Qing Cai,

East China Normal University, China

Reviewed by:

Zhong-Lin Lu,

New York University, United States

Linjun Zhang,

Beijing Language and Culture

University, China

*Correspondence:

Caicai Zhang

caicai.zhang@polyu.edu.hk

Specialty section:

This article was submitted to

Language Sciences,

a section of the journal

Frontiers in Psychology

Received: 02 February 2021 Accepted: 09 July 2021

Published: 29 July 2021

Citation:

Zhang Y, Pattamadilok $C$, Lau DK-Y, Bakhtiar M, Yim L-Y,

Leung K-Y and Zhang C (2021) Early

Auditory Event-Related Potentials Are

Modulated by Alphabetic Literacy

Skills in Logographic Chinese

Readers. Front. Psychol. 12:663166.

doi: 10.3389/fpsyg.2021.663166

\section{Early Auditory Event-Related Potentials Are Modulated by Alphabetic Literacy Skills in Logographic Chinese Readers}

\author{
Yubin Zhang ${ }^{1}$, Chotiga Pattamadilok², Dustin Kai-Yan Lau ${ }^{3}$, Mehdi Bakhtiar \\ Long-Ying Yim ${ }^{3}$, Ka-Yui Leung ${ }^{3}$ and Caicai Zhang ${ }^{3,5 *}$
}

'Department of Linguistics, University of Southern California, Los Angeles, CA, United States, ${ }^{2}$ Laboratoire Parole et Langage (LPL), CNRS, Aix Marseille University, Aix-en-Provence, France, ${ }^{3}$ Department of Chinese and Bilingual Studies, The Hong Kong Polytechnic University, Hong Kong, China, ${ }^{4}$ Unit of Human Communication, Development, and Information Sciences, The University of Hong Kong, Hong Kong, China, ${ }^{5}$ Research Centre for Language, Cognition, and Neuroscience, The Hong Kong Polytechnic University, Hong Kong, China

The acquisition of an alphabetic orthography transforms speech processing in the human brain. Behavioral evidence shows that phonological awareness as assessed by meta-phonological tasks like phoneme judgment, is enhanced by alphabetic literacy acquisition. The current study investigates the time-course of the neuro-cognitive operations underlying this enhancement as revealed by event-related potentials (ERPs). Chinese readers with and without proficiency in Jyutping, a Romanization system of Cantonese, were recruited for an auditory onset phoneme judgment task; their behavioral responses and the elicited ERPs were examined. Proficient readers of Jyutping achieved higher response accuracy and exhibited more negative-going ERPs in three early ERP time-windows corresponding to the P1, N1, and P2 components. The phonological mismatch negativity component exhibited sensitivity to both onset and rhyme mismatch in the speech stimuli, but it was not modulated by alphabetic literacy skills. The sustained negativity in the P1-N1-P2 time-windows is interpreted as reflecting enhanced phonetic/phonological processing or attentional/awareness modulation associated with alphabetic literacy and phonological awareness skills.

Keywords: phonological awareness, alphabetic orthography, auditory onset phoneme judgment, event-related potentials, Chinese

\section{INTRODUCTION}

The functional organization of the human brain can accommodate itself to new experiences. As a recent cultural invention in human history, the writing system of language influences how the brain represents and processes phonological information in a significant way (Castro-Caldas et al., 1998; Perre et al., 2009; Dehaene et al., 2010, 2015; Monzalvo and Dehaene-Lambertz, 2013; Morais, 2021).

One example of such influence is that the grain size of phonological representations and processing has been suggested to be shaped by the units employed in the orthographic system (Ziegler and Goswami, 2005). In an alphabetic writing system such as English, Dutch or French, the 
orthographic letters represent speech sounds at a more finegrained phonemic level. However, in a logographic system like Chinese, the characters are associated with phonology at a much coarser grain size, i.e., the morpho-syllabic level (Perfetti et al., 2010). Although a significant portion of compound Chinese characters contains phonetic radicals that can be employed for retrieving phonological information, the phonetic radicals refer to the pronunciation of the whole syllable instead of individual phonemes (Perfetti and Tan, 1998). Phonological awareness and representations at the relatively fine-grained abstract phonemic level have been argued to be heavily influenced by the acquisition of an alphabetic writing system. Some researchers even argue that the phoneme is a literate cognitive concept induced by alphabetic literacy acquisition (Morais et al., 1979; Read et al., 1986; Morais, 2021). Now, prior to the introduction of the current study, we turn to a brief overview of previous neural and behavioral evidence on the influences of learning an alphabetic writing system on spoken language representations and processing.

\section{The Impact of Acquiring an Alphabetic Script on Speech Representations and Processing: Behavioral and Neural Evidence}

The most prevalent behavioral paradigms to investigate speakers' representations and awareness to phonological units are tasks that tap meta-phonological skills, such as phoneme deletion/addition (Morais et al., 1979; Read et al., 1986; de Gelder et al., 1993), pseudoword repetition (Reis and CastroCaldas, 1997), auditory onset/rhyme judgment (de Gelder et al., 1993), sound matching or detection (Ho and Bryant, 1997; Cheung et al., 2001), and phoneme counting (Liberman et al., 1974). Performance on the identification and manipulation of phonological units in such tasks is considered as an index of phonological awareness to speech units with different grain sizes. Speakers' phonemic awareness is evaluated when the task focuses on phonemic manipulation.

Earlier behavioral evidence showed that compared with proficient readers of an alphabetic script, preliterate children have difficulties with explicit phoneme manipulation, such as counting the number of phonemes in speech stimuli (Liberman et al., 1974). However, this difference in performance could also be due to differences in the maturation of general cognitive skills between the two populations.

Later work investigated phonological awareness in literate and illiterate adults in an alphabetic script (Morais et al., 1979; Reis and Castro-Caldas, 1997). Similar difficulties with explicit phonemic analysis have been observed in illiterate adults even though their speech and general cognitive systems have reached maturity. Illiterates were found to show lower performance than their literate peers in tasks tapping explicit phonemic analysis, such as phoneme addition/deletion (Morais et al., 1979) and pseudoword repetition (Reis and Castro-Caldas, 1997).

Another line of behavioral evidence comes from studies that focus on phonological awareness in readers with different levels of knowledge of logographic versus alphabetic scripts (Read et al., 1986; de Gelder et al., 1993; Ho and Bryant, 1997;
Cheung et al., 2001; Shu et al., 2008). In an early study, Read et al. (1986) reported that Chinese readers who were literate only in the logographic script exhibited poorer phonemic awareness than readers with additional knowledge of Pinyin, which is an alphabetic script of Mandarin Chinese. More recently, Shu et al. (2008) investigated the development of phonological awareness in young Chinese children in a series of sound detection tasks. Their results showed that the kindergarten children could not perform the onset phoneme detection task above chance level whereas the first graders with Pinyin training achieved 70\% accuracy, suggesting that some levels of phonological awareness especially onset phonemic awareness is dependent on alphabetic literacy. However, since this study did not include first graders without Pinyin instruction, we cannot completely exclude the possibility that the better performance in onset detection for the first graders was due to their better general cognitive skills and more experience with the ambient spoken language. In a crosslinguistic developmental study, Ho and Bryant (1997) found that Cantonese-speaking Chinese children in Hong Kong developed consonant awareness later than their English counterparts in primary school. Cheung et al. (2001) compared the phonological awareness skills of Hong Kong Cantonese-speaking children who had knowledge of the logographic Chinese script only and Guangzhou Cantonese-speaking children who received training in both logographic Chinese and Pinyin scripts. They found that Guangzhou children performed better than their Hong Kong counterparts in onset and coda matching, suggesting the influences of alphabetic knowledge on phonemic awareness.

Further behavioral evidence comes from other behavioral paradigms, like word-form encoding in production and primednaming experiments in the visual modality (Perfetti and Bell, 1991; Perfetti and Tan, 1998; Chen et al., 2002). These experiments do not require explicit phonemic manipulation but still provide insight on the cross-linguistic differences in the grain size of phonological representations associated with orthographic systems. In an implicit priming study on wordform encoding in Chinese, Chen et al. (2002) found that both syllable+tone and syllable-alone units evoked implicit priming effects, whereas the syllable onset unit (corresponding to a phoneme) did not. In contrast, onset priming was detected in Dutch speakers using a similar paradigm (Meyer, 1991). The discrepancy suggests phonemic level encoding in Dutch but not in Chinese (Chen et al., 2002). In Chinese character primed-naming experiments, Perfetti and Tan (1998) reported that facilitatory homophonic (phonological) priming effects, e.g., for a prime-target pair like “其 (Pinyin: qí) - 齐(qí)," occurred relatively late, when the early facilitatory graphic priming effects, e.g., “何 (hé) - 向 (xiàng)," became inhibitory. The authors suggested that in Chinese readers, graphic and phonological information are activated independently in distinct cycles, and phonological activation arises once orthographic processing has been completed, presumably without phonemic assembly. These results are in contrast to those obtained from an earlier priming study in English readers (Perfetti and Bell, 1991). In that study, the target word (e.g., rate), was presented visually after three types of pseudoword primes-phonemic prime (e.g., rait), graphemic 
prime (e.g., ralt), and control prime (e.g., busk). The results revealed early and synchronously increasing facilitatory effects of both homophonic and graphic primes, thus suggesting that phonological activation arises synchronously with the processing of the constituent graphic units.

Neuroimaging studies using tasks that require metaphonological skills have revealed multiple neuro-cognitive networks that are sensitive to alphabetic and phonological awareness skills (Castro-Caldas et al., 1998; Burton et al., 2000; Petersson et al., 2000; Brennan et al., 2013). The leftdominant peri-sylvian language network, including the inferior frontal gyrus (IFG), superior temporal gyrus (STG), and planum temporale (PT) that are suggested to be responsible for phonetic/phonological processing, has been frequently found to be implicated in tasks that require meta-phonological analysis and literacy-induced changes in speech and language processing (Castro-Caldas et al., 1998; Burton et al., 2000; Petersson et al., 2000; Dehaene et al., 2010, 2015; Brennan et al., 2013; Monzalvo and Dehaene-Lambertz, 2013). Moreover, the attentional network involving the anterior cingulate cortex (ACC) and the central executive network including the prefrontal cortex, have been shown to be engaged in such tasks, and to vary in activation levels according to alphabetic literacy and phonological awareness skills (Castro-Caldas et al., 1998; Burton et al., 2000; Petersson et al., 2000).

Burton et al. (2000) conducted an fMRI study to examine the contribution of the frontal cortices during auditory phoneme judgment under different phonemic segmentation requirements. Participants were asked to perform "same/different" judgment on the onset consonants of English word pairs presented in two lists- "global" and "segmental". In the global list, where the two words in a pair always shared the same rhyme in a word pair (e.g., dip-tip and dip-dip), participants were expected to adopt a "global strategy" that consists in making judgments without segmenting out the onset phonemes. Conversely, in the segmental list where the rhymes differed (e.g., dip-tomb and dip-doom), separating the onset phonemes from the rest of the words was assumed to be mandatory. The imaging results indicated that the STG, subserving phonetic/phonological processing, was activated for both lists. However, only the segmental list elicited activations in frontal cortices (left IFG and middle frontal gyrus), which the authors interpreted as increased demands of phonemic segmentation, articulatory recoding or working memory in the segmental list. Stronger ACC activations were also found in the segmental list. This result possibly reflected increased attentional modulation or conflict monitoring because more attentional resources might be needed in the segmental list to focus on the target onset dimension and to ignore or inhibit the irrelevant rhyme differences in a word pair.

A recent fMRI study by Brennan et al. (2013) investigated the developmental differences in the phonological network in Chinese and English speakers using an auditory rhyme judgment task. The imaging results revealed developmental increase in the activation of the left STG, IFG, and inferior parietal lobule (IPL) for English speakers only. This is taken as evidence that literacy acquisition leads to the reorganization of the phonological network for speakers of a language with an alphabetic writing system only.

\section{The Current Study}

While there is a large body of literature examining the neurocognitive networks in meta-phonological tasks, the temporal dynamics of these cognitive operations underlying phonological awareness skills during phoneme judgment is still unclear. More importantly, it remains to be studied how the timecourse of these neuro-cognitive events in phonemic processing can be influenced by the acquisition of an alphabetic script. Existing ERP studies using meta-phonological tasks were not designed specifically for this question (McPherson et al., 1998; Pattamadilok et al., 2011; Lafontaine et al., 2012). For example, Lafontaine et al. (2012) employed an auditory onset judgment task and manipulated the congruency between the phonological and orthographic information of the onsets in a French word pair. Their goal was to investigate the time-course of phonological and orthographic activations. As such, there remain research gaps regarding the ERP indices of how learning an alphabetic system affects the time-course of the neuro-cognitive operations underlying phonological awareness skills required in metaphonological tasks.

The major goal of the present ERP study is to identify the speech processing stages and the corresponding neuro-cognitive operations involved in phonemic processing that are sensitive to alphabetic literacy skills in adult readers of logographic Chinese. To this end, we recruited two groups of Cantonese speakers with and without proficiency in Jyutping, a romanization system of spoken Cantonese, for an auditory onset judgment task. One motivation for choosing Jyutping is that it allows us to investigate whether and to what extent the relatively late acquisition of an alphabetic script influences the neuro-cognitive processes underpinning phonological awareness skills in adult readers of logographic Chinese. In Mainland China, Pinyin is taught to children at the same time as they begin to learn the Chinese logographic writing system. However, Cantonese children in Hong Kong learn the logographic Chinese writing system without the assistance of a native alphabetic script. Some Cantonese speakers choose to learn Jyutping primarily for the purpose of inputting Chinese on social media. Although most Cantonese speakers in Hong Kong have some acquaintance with the alphabetic English script, it remains controversial whether learning to read in an alphabetic script of a second language like English would influence their phonological processing in Cantonese in the same way and to the same extent as acquiring an alphabetic script of their native language Cantonese (Bialystok et al., 2005; Dodd et al., 2008; Deng et al., 2019).

Besides the between-subject manipulation of Jyutping knowledge (i.e., the group factor), the speech stimuli employed in the auditory onset phoneme judgment task were divided into two lists-global and segmental-as in Burton et al. (2000) to manipulate the segmentation demands. The paired Cantonese pseudoword stimuli in the global list always contained the same rhyme (e.g., $\mathrm{paa}^{2}-\mathrm{paa}^{2}$ and $\mathrm{paa}^{2}-\mathrm{faa}^{2}$, transcribed in Jyutping) whereas the stimuli in the segmental list contained different rhymes (e.g., $\mathrm{paa}^{2}$-paai ${ }^{2}$ and $\mathrm{paa}^{2}$-faai ${ }^{2}$ ). This list manipulation 
allowed us to test whether the alphabetic knowledge affects speech processing situations with different "segmentation" demands in the same way (Burton et al., 2000). Finally, for the purpose of the same/different onset phoneme judgment task, within each list, pairs of stimuli with both congruent and incongruent onsets were included in the materials. Altogether, these manipulations led to a $2 \times 2 \times 2$ design with three factors: group (non-Jyutping vs. Jyutping), list (global vs. segmental) and onset congruency (congruent vs. incongruent).

For behavioral responses, we predicted that the group with Jyutping proficiency would achieve higher accuracy and respond more quickly than the group with low Jyutping proficiency (Read et al., 1986; de Gelder et al., 1993; Ho and Bryant, 1997; Cheung et al., 2001; Shu et al., 2008). Furthermore, the segmental list would elicit less accurate and slower responses than the global list (Burton et al., 2000). The group effect could also be amplified by task difficulties caused by the characteristics of the list or onset congruency.

Regarding the predictions on ERP responses, a growing number of studies have shown that more efficient auditory or speech/musical processing abilities are associated with an enhancement of early auditory evoked potentials like N1-P2 (Tremblay et al., 2001; Tremblay and Kraus, 2002; Baumann et al., 2008; Shahin, 2011). Since acquiring an alphabetic script leads to the improvement of speech processing abilities (Read et al., 1986; Castro-Caldas et al., 1998; Brennan et al., 2013; Dehaene et al., 2015), we could reasonably expect the participants with Jyutping expertise to show higher amplitude in the N1 and P2 components than those without Jyutping proficiency. Additionally, the impact of the knowledge of a native alphabetic script might also be found at a later phonological processing stage as indexed by the phonological mismatch negativity (PMN). A prevalent view is that the PMN reflects the stage of translating acoustic properties of speech sounds into phonemic candidates, and this process is affected by the mismatch between phonemic expectations in the working memory established by prior contexts and the actually presented speech signals (Connolly and Phillips, 1994; Newman et al., 2003; Lafontaine et al., 2012; Zhang, 2018). In the auditory phoneme judgment task, the PMN component would reflect an active detection of a mismatch in phonological units, like onsets and rhymes, in a pair of speech stimuli (Lafontaine et al., 2012). For example, in our design, two types of phonological mismatch would be expected-one induced by the manipulation of onset congruency, which was the target dimension of the task, and the other induced by the list manipulation, which might engender additional phonological mismatch at the rhyme level. If the PMN is modulated by alphabetic literacy skills, the ERP amplitude in this time-window would be affected by the interaction between group and list or between group and onset congruency.

\section{METHODS}

\section{Participants}

Twenty-four logographic Chinese readers with Jyutping competency (henceforth, the Jyutping group) and twenty-two participants without Jyutping competency (henceforth, the
non-Jyutping group) participated in the study. The two groups of participants were determined based on their Jyutping proficiency, which was evaluated by a timed Jyutping transcription test. The Jyutping test included 20 disyllabic Chinese words presented to the participants as 40 Chinese characters, which covered as many onsets and rhymes in Cantonese as possible. Participants were required to transcribe the 40 Chinese characters into Jyutping labels within 2 minutes (e.g., from “變化” to $\mathrm{bin}^{3}$ $\mathrm{faa}^{3}$ ). In line with the aim of the study, participants' scores for transcribing onset consonants were calculated (40 points in total). The Jyutping group obtained much higher average scores for onset phoneme transcription than the non-Jyutping group (mean \pm SD: $29.7 \pm 8.3$ vs. $7.5 \pm 3.7$ ). In both groups, the majority of the participants had completed or had been pursuing a college degree at the time of participation and none of them majored in linguistics or psychology. The two groups were matched for age, English proficiency (standardized English scores), and musical experience. Their English proficiency was evaluated based on their most recent scores obtained in standardized English tests used in Hong Kong (IELTS, HKDSE-ENG, or HKALE-ENG). Most of the participants were right-handed and all of them had no reported hearing, speech, or language disorders. The detailed demographic information of the two groups is presented in Table 1.

Informed written consent was obtained from the participants in compliance with the experiment protocols approved by the Human Subjects Ethics Sub-committee of the Hong Kong Polytechnic University and with the 1964 Helsinki declaration and its later amendments or comparable ethical standards.

\section{Materials}

The auditory phoneme judgment task included 96 Cantonese pseudoword pairs in spoken forms, which were constructed

TABLE 1 | Participant information.

\begin{tabular}{lcc}
\hline & Jyutping & Non-Jyutping \\
\hline Total & 24 & 22 \\
Gender (number of male/female) & $17 / 7$ & $13 / 9$ \\
Age (mean \pm SD) & $22.4( \pm 4.2)$ & $21.9( \pm 2.8)$ \\
Jyutping scores (mean \pm SD; total: 40) & $29.7( \pm 8.3)$ & $7.5( \pm 3.7)$ \\
Standardized English scores ${ }^{1}$ (mean \pm SD) & $6.8( \pm 0.6)$ & $6.5( \pm 0.6)$ \\
Musical training ${ }^{2}$ (No. of participants) & 3 & 3 \\
Education level (No. of participants with a & 22 & 19 \\
bachelor's degree or higher) $^{\text {Handedness (No. of right-handed participants) }}$ & 23 & 22 \\
\hline
\end{tabular}

${ }^{1}$ A participant's standardized English score was calculated from the International English Language Testing System (IELTS), Hong Kong Diploma of Secondary Education Examination-English Language (HKDSE-ENG), or Hong Kong Advanced Level Examination-English Language (HKALE-ENG), respectively. To make the standardized English tests scores comparable across participants, the HKDSEENG and HKALE-ENG scores were converted into equivalent IELTS band scores based on the results of a benchmarking study comparing IELTS and HKDSE-ENG/HLALE-ENG by the Hong Kong Examinations and Assessment Authority (HKEAA).

${ }^{2}$ Participants who have received musical training in either musical instrument or vocal singing for 3 years or longer were considered to be participants with musical training. 
from six sets of Cantonese pseudowords (see Table 2). Each set contained four monosyllabic pseudowords, which are phonotactically plausible syllables, but their combinations with certain tones do not yield real words. For example, for the pseudoword "paai"," "paai" is a legal Cantonese syllable, but combining this syllable with Tone 2 (high-rising tone) generates a pseudoword. Only Tone 1 (high-level tone), Tone 2 (highrising tone), and Tone 4 (low-falling tone) were included as they are the most easily recognizable tones in Cantonese (Peng et al., 2012). Within each set, four syllables (e.g., paa ${ }^{2}, \mathrm{paai}^{2}$, $\mathrm{faa}^{2}$, and faai ${ }^{2}$ ) were formed by combining two onsets (e.g., $\mathrm{p}$ and f) and two rhymes (e.g., aa and aai), with the tone (e.g., tone 2) kept identical. For each of the six sets, 16 pseudoword pairs were constructed by combining each pseudoword with itself and then with the other three pseudowords (e.g., combining paa ${ }^{2}$ with other pseudowords-paa ${ }^{2}-$ paa $^{2}$, paa $^{2}-$ paai $^{2}$, paa $^{2}-\mathrm{faa}^{2}$, and $\mathrm{paa}^{2}-\mathrm{faai}^{2}$ ), resulting in a total of 96 pairs of pseudowords for all the six sets. The materials differed in two aspects-list (or rhyme difference, global vs. segmental) and onset congruency (onset difference, congruent vs. incongruent). Following Burton et al. (2000), the resulting 96 pseudoword pairs were divided into two lists, representing two experimental conditions-"global" and "segmental". In the global list (48 pairs), two pseudowords in a pair had identical rhymes, whereas the onsets could be either congruent (e.g., paa $^{2}-\mathrm{paa}^{2}$, paai $^{2}-$ paai $^{2}, \mathrm{faa}^{2}-\mathrm{faa}^{2}$, and faai $^{2}-$ faai $^{2}$ ) or incongruent $\left(\mathrm{paa}^{2}-\mathrm{faa}^{2}, \mathrm{faa}^{2}-\mathrm{paa}^{2}\right.$, paai $^{2}-\mathrm{faai}^{2}$, and faai $^{2}$-paai ${ }^{2}$ ). In the segmental list (48 pairs), two pseudowords had different rhymes, whereas again, the onsets could be either congruent (e.g., paa $^{2}$-paai ${ }^{2}$, paai ${ }^{2}-$ paa $^{2}$, faa $^{2}-$ faai $^{2}$, and faai ${ }^{2}-\mathrm{faa}^{2}$ ) or incongruent (paa ${ }^{2}-\mathrm{faai}^{2}, \mathrm{faai}^{2}-\mathrm{paa}^{2}, \mathrm{faa}^{2}-\mathrm{paai}^{2}$, and $\mathrm{paai}^{2}-$ $\mathrm{faa}^{2}$ ). The speech materials were recorded in a sound-attenuated booth by a female Cantonese speaker. The duration and mean intensity of the stimuli were normalized to $560 \mathrm{~ms}$ and $70 \mathrm{~dB}$, respectively. Within each pseudoword pair, the inter-stimulus interval (ISI) was $440 \mathrm{~ms}$.

\section{Procedure}

Participants were seated in an electrically-shielded booth. EEG signals were continuously recorded and digitized at a sampling rate of 2,048 $\mathrm{Hz}$ using a 32-channel ActiveTwo EEG system (BioSemi B. V., Amsterdam, Netherlands). The whole experiment was divided into two blocks, representing the global and segmental lists. The auditory speech stimuli were repeated twice, generating 96 trials for each list. A fixation cross, indicating the beginning of a trial, was presented at the center of the screen, and remained until the end of a trial. A pseudoword pair was presented binaurally through inserted earphones $2000 \mathrm{~ms}$ after fixation cross onset. Within each block, the auditory stimuli were presented pseudo-randomly. The participants were required to judge whether the two auditory speech stimuli in a pair shared the same onset by pressing the left or right button of the response box with their left or right index finger without a time limit. A manual response terminated a trial and the procedure proceeded to the next trial. The inter-trial interval was jittered between 1600 and $1800 \mathrm{~ms}$.

\section{Data Analysis}

The response accuracy and reaction time (measured from the onset of the second stimulus in a pair) were analyzed by a generalized linear mixed-effects model and a general linear mixed-effects model, respectively, using the lme4 package in $R$ (Bates et al., 2020). The raw reaction time (RT) was logtransformed to achieve closer approximation of normality of residuals. Both models included three deviation-coded $(-0.5$, 0.5) categorical variables-group (non-Jyutping vs. Jyutping), list (global vs. segmental), onset congruency (congruent vs. incongruent)-and their interactions as fixed effects. For the random-effects structures of both models, we began by including the by-participant random intercept only. Then, we tested whether including the other by-participant random slopes significantly improved model fit by the log-likelihood ratio test. According to the test results, the random slopes of list, onset congruency and their interaction were justified for both models. When an interaction effect was found, we also conducted posthoc analyses using the emmeans package (Lenth et al., 2009).

The raw EEG data were preprocessed using EEGLab (version 2019). The raw EEG data were first band-pass filtered (0.1$30 \mathrm{~Hz}$ ) and then segmented into $600 \mathrm{~ms}$ epochs, time-locked to the onset of the second pseudoword of a stimulus pair. The baseline was $100 \mathrm{~ms}$ before the onset of the second stimulus in a pseudoword pair and the post-onset interval was $500 \mathrm{~ms}$. Baseline-correction was applied according to the $100 \mathrm{~ms}$ prestimulus interval. The epoched data were recalculated against the average-mastoid reference. Prior to Independent Component Analysis (ICA), we pruned bad epochs with non-stereotyped or paroxysmal artifacts which would affect the quality of ICA decomposition (Onton et al., 2006). This step led to a loss of $4.56 \%$ of the trials. After pruning, ICA was performed on the remaining data using the extended informax algorithm (Delorme and Makeig, 2004). Artifactual components, reflecting eye blinks and eye movements, were manually removed by inspecting the ICA topographies, waveforms and spectra. To calculate

TABLE 2 | Six sets of auditory Cantonese pseudowords transcribed in Jyutping and IPA (in brackets).

\begin{tabular}{|c|c|c|c|c|c|c|c|c|}
\hline Set 1 & aa (/a:/) & aai (/a:i/) & Set 2 & ei (/ei/) & eoi (/ey/) & Set 3 & aau (/a:u/) & $\mathrm{i}(/ \mathrm{i}: /)$ \\
\hline $\mathrm{p}\left(/ \mathrm{p}^{h} /\right)$ & paa2 & paai2 & $d(/ t /)$ & dei4 & deoi4 & $\mathrm{m}(/ \mathrm{m} /)$ & maau2 & $\mathrm{mi} 2$ \\
\hline$f(/ f /)$ & faa2 & faai2 & $g(/ k /)$ & gei4 & geoi4 & $\mathrm{I}(/ / /)$ & laau2 & li2 \\
\hline Set 4 & it (/i:t/) & aat (/a:t/) & Set 5 & in (/i:n/) & eng $(/ \varepsilon: \eta /)$ & Set 6 & ek (/ع:k/) & ok (/っ:k/) \\
\hline$c\left(/ \mathrm{ts}^{h} /\right)$ & cit1 & caat1 & $g(/ k)$ & gin4 & geng4 & $t\left(/ t^{h} /\right)$ & tek1 & tok1 \\
\hline I (/I/) & lit1 & laat1 & $\mathrm{h}(/ \mathrm{h} /)$ & hin4 & heng4 & $\mathrm{s}(/ \mathrm{s} /)$ & sek1 & sok1 \\
\hline
\end{tabular}


ERPs, the resulting epochs were averaged for each participant and each condition.

For the ERP analysis, we first calculated the global field power (GFP, i.e., spatial root mean square of voltage over all the electrodes) on the grand-averaged waveforms averaged across all the participants and conditions according to the following formula:

$$
\mathrm{GFP}_{t}=\sqrt{\frac{\sum_{i=1}^{k} V_{i t}^{2}}{k}}
$$

Where $t$ is time, $k$ is the total number of channels $(k=32), V_{i t}$ is the voltage at channel $i$ and time $t$.

Based on the GFP and previous ERP literature (Connolly and Phillips, 1994; Tremblay and Kraus, 2002; Martin et al., 2008; Zhang, 2018), we analyzed the ERPs in four time-windowsP1 (0-93 ms), N1 (95-237 ms), P2 (239-353 ms), and PMN (355-500 ms, see Figure 1). ERP amplitude in the P1-N1-P2 time-window was analyzed by averaging the ERP waves from a fronto-central electrode cluster (F3, Fz, F1, FC1, FC2, and Cz; Näätänen and Picton, 1987; Tremblay and Kraus, 2002; Martin et al., 2008). The PMN analysis was based on the averages of ERPs from a centro-parietal electrode cluster (P3, Pz, P4, CP1, CP2, and Cz; Lafontaine et al., 2012). Since we did not predict hemispheric laterality effects, the electrodes were not further divided into left, midline, and right sites. Mean ERP amplitude of each time-window was calculated for each participant, list, and onset congruency condition, and was submitted to a threeway ANOVA, with three factors group, list and onset congruency. The ANOVA was conducted using the afex package in $R$ (Singmann et al., 2020). When an interaction effect was detected, post-hoc analyses were conducted using the emmeans package (Lenth et al., 2009).

\section{RESULTS}

\section{Behavioral Results}

The behavioral results are displayed in Figure 2. The accuracy model revealed a significant main effect of group $(\beta=0.67$, $z=2.47, p<0.01)$. As expected, the Jyutping group achieved higher accuracy than the non-Jyutping group. Additionally, there was a significant main effect of list $(\beta=-0.88, z=-5.13$, $p<0.001$ ), with more accurate responses elicited in the global list than in the segmental list. A significant main effect of onset congruency was also found $(\beta=1.14, z=5.49, p<0.001)$. There were generally more accurate responses for trials with incongruent onsets than congruent onsets, but the effect of onset congruency was further modulated by list, as shown by a significant interaction between list and onset congruency $(\beta=2.07, z=5.72, p<0.001)$. Post-hoc analysis revealed more accurate responses for the global list than the segmental list in trials with congruent onsets only $(z=-10.88, p<0.001)$. The results showed that the congruent onset/segmental list condition elicited the least accurate responses. The interactions between group and other factors were not significant $(p s>0.05)$.

The RT model revealed a significant main effect of list ( $\beta=0.03, t=4.23, p<0.001)$, reflecting generally faster responses to the global list than to the segmental list, which is consistent with the accuracy results and confirmed our prediction that performing phoneme judgment for the segmental list is more challenging. This effect was modulated by onset congruency $(\beta=-$ $0.04, t=-6.4, p<0.001)$. Post-hoc analysis revealed significantly faster responses for the global list than the segmental list in trials with congruent onsets only $(z=6.31, p<0.001)$, which is similar to the pattern reported above on accuracy. No significant group effect or its interactions with other factors were found ( $p s>0.05$ ).

\section{ERP Results}

For auditory P1-N1-P2, which was analyzed based on the frontocentral electrode cluster, the ANOVAs revealed that the Jyutping group showed significantly more negative-going ERPs than the non-Jyutping group in $\mathrm{P} 1[F(1,44)=12.42, p<0.01], \mathrm{N} 1[F(1$, $44)=11.21, p<0.01]$, and P2 time-windows $[F(1,44)=8.42$, $p<0.01]$. There was also a significant interaction between group and onset congruency in the P1 time-window $[F(1,44)=6.04$, $p<0.05]$. Post-hoc analysis with Holm-Bonferroni correction revealed that the Jyutping group exhibited more negative-going ERPs than the non-Jyutping group in the P1 time-window for congruent trials $(t=-4.29, p<0.001)$, but not for incongruent trials $(t=-1.71, p=0.09)$. Furthermore, the segmental list elicited more negative-going ERPs than the global list in the P2 timewindow only $[F(1,44)=5.85, p<0.05]$. The effects revealed by ANOVAs are displayed in Figure 3. The ERP waveforms averaged across all the selected electrodes in the fronto-central cluster and the topographic maps of the ERPs in the P1-N1-P2 time windows pooled across congruent and incongruent onsets are illustrated in Figure 4. Overall, the group effect seems to manifest as a sustained negativity that extends from the P1 to the P2 time-window.

For the PMN component which was analyzed based on the centro-parietal electrode cluster, there was a significant main effect of list $[F(1,44)=15.15, p<0.001]$, with larger negativity elicited in the segmental list than in the global list. We also found a significant effect of onset congruency $[F(1$, $44)=28.31, p<0.001]$, with larger negativity elicited by trials with incongruent onsets than with congruent onsets. No significant effect of group or its interactions with other factors were found $(p s>0.05$ ). Thus, there is no evidence that the phonological mismatch detection process is modulated by the participants' knowledge of Jyutping. The onset congruency effect reflected the mismatch between the onsets of the pseudowords in a pair. Furthermore, the list effect might reflect the detection of rhyme mismatch, even though the task focused on onset judgment. To further explore the PMN pattern, post-hoc pairwise comparisons with Holm-Bonferroni correction were performed among the four conditions collapsed across Jyutping and non-Jyutping groups, that is, the four combinations of onset and rhyme mismatch-incongruent onset/segmental list (different onsets and different rhymes), congruent onset/segmental list (same onset and different rhymes), incongruent onset/global list (different onsets and same rhyme) and congruent onset/global list (same onset and same rhyme). Compared with the congruent onset/global list condition, the other three conditions exhibited more negative ERPs in the PMN time-window ( $p s<0.001)$. These results reaffirmed our prediction that PMN would be 


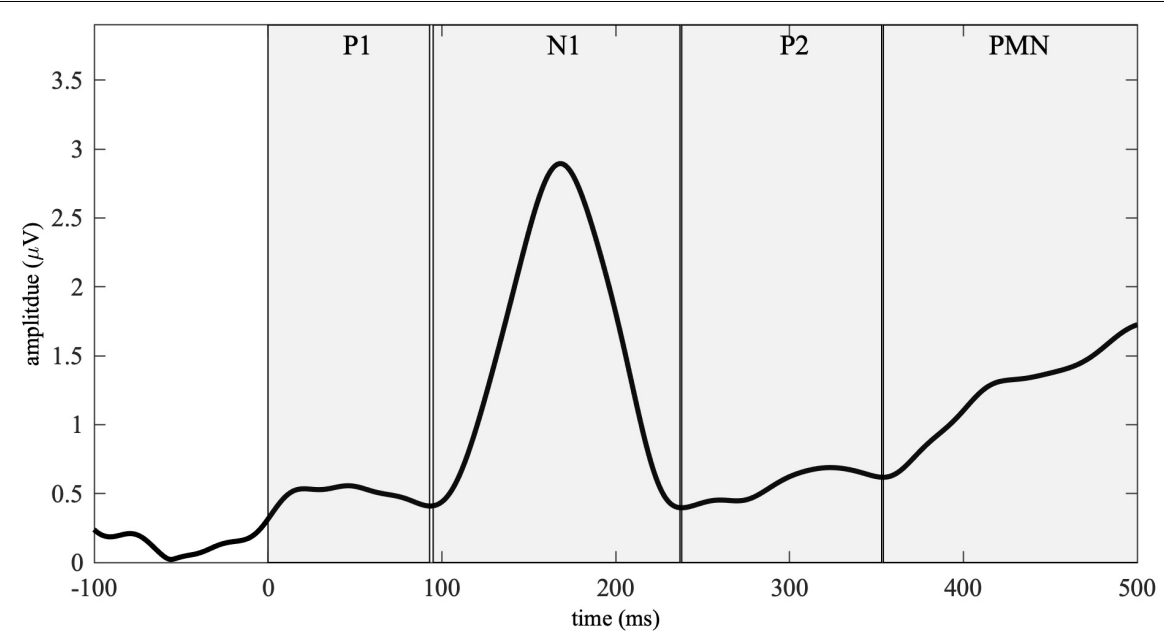

FIGURE 1 | Global field power (GFP) calculated from the grand-averaged waveforms across all the participants and conditions. Four event-related potential (ERP) time-windows- (0-93 ms), N1 (95-237 ms), P2 (239-353 ms), and phonological mismatch negativity (PMN, 355-500 ms) were identified based on the GFP. The boundaries across time-windows $(94,238$, and $354 \mathrm{~ms}$ ) were defined as the minimal values from 50-150, 200-300, and 300-400 ms.

A The group effect on accuracy

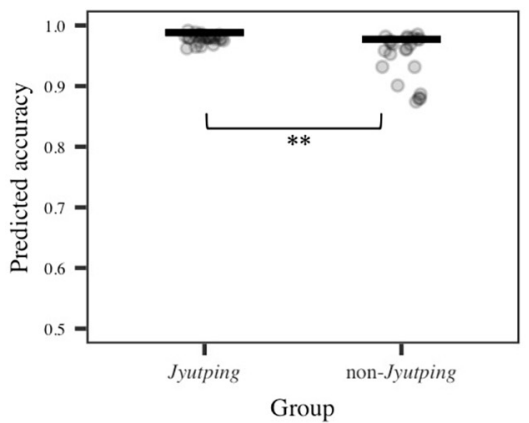

B

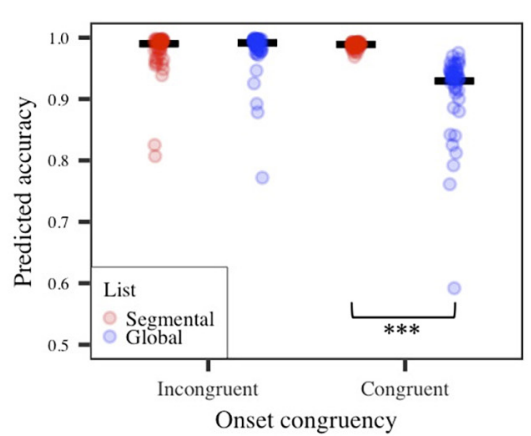

C

List and onset congruency effects on RT

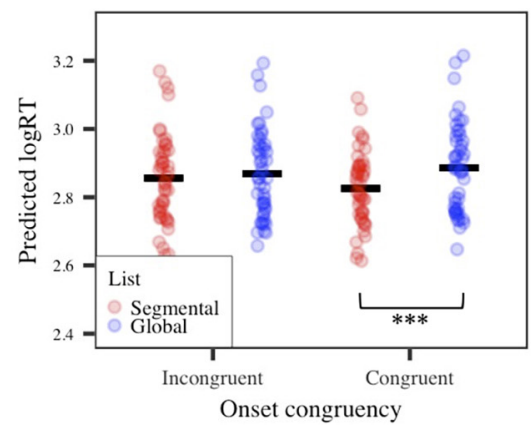

FIGURE 2 | Accuracy and reaction time (RT) results. (A) The group effect on accuracy. (B) List and onset congruency effects on accuracy. (C) List and onset congruency effects on RT. The solid line denotes estimated means based on fixed effects while each dot represents individual fitted values. Significance levels: ${ }^{*} p<0.05 ;{ }^{* *} p<0.01 ;{ }^{* \star *} p<0.001$.

elicited for both onset and rhyme mismatches. Moreover, the incongruent onset/segmental list condition elicited larger negativity than the congruent onset/segmental list condition ( $t=-4.03, p<0.001)$, and the incongruent onset/global list condition $(t=-3.39, p<0.01)$. This result suggested the largest PMN in the incongruent onset/segmental list condition with both onset and rhyme mismatches. However, the congruent onset/segmental list condition, and incongruent onset/global list condition did not differ from each other significantly $(t=0.25$, $p=0.81$ ), suggesting similar PMN amplitude for rhyme and onset mismatch. Thus, the additional analyses indicated cumulative effects of onset and rhyme mismatch on the PMN amplitude (least-square means in four conditions: congruent onset/global list: $4.04 \mu \mathrm{V}$; incongruent onset/global list: $2.33 \mu \mathrm{V}$; congruent onset/segmental list: $2.19 \mu \mathrm{V}$; incongruent onset/segmental list: $0.40 \mu \mathrm{V})$. The PMN effects revealed by ANOVAs are displayed in Figure 5. The ERP waveforms averaged across all the selected electrodes in the centro-parietal cluster are displayed in
Figure 6A. The topographic maps of the three isolated PMNs induced by onset mismatch, rhyme mismatch and rhyme+onset mismatch are displayed in Figure 6B. As indicated by the topographic maps, the PMN has a centro-parietal distribution and, consistent with the statistical results, it has the largest amplitude in the rhyme+onset mismatch condition. However, no group differences can be observed.

\section{DISCUSSION}

We found that participants in the Jyutping group achieved higher accuracy than the non-Jyutping group in auditory onset phoneme judgment. This behavioral result is associated with the ERP pattern that the Jyutping group showed more negative-going ERPs in early auditory P1-N1-P2 than the nonJyutping group. These findings provide further support for the claim that speech representations and processing at the 
A

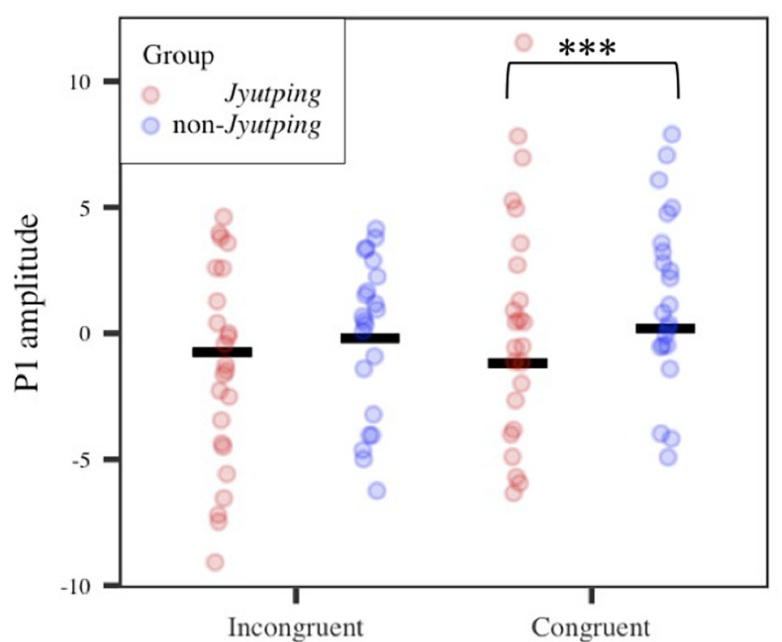

Onset congruency

C The group effect on P2

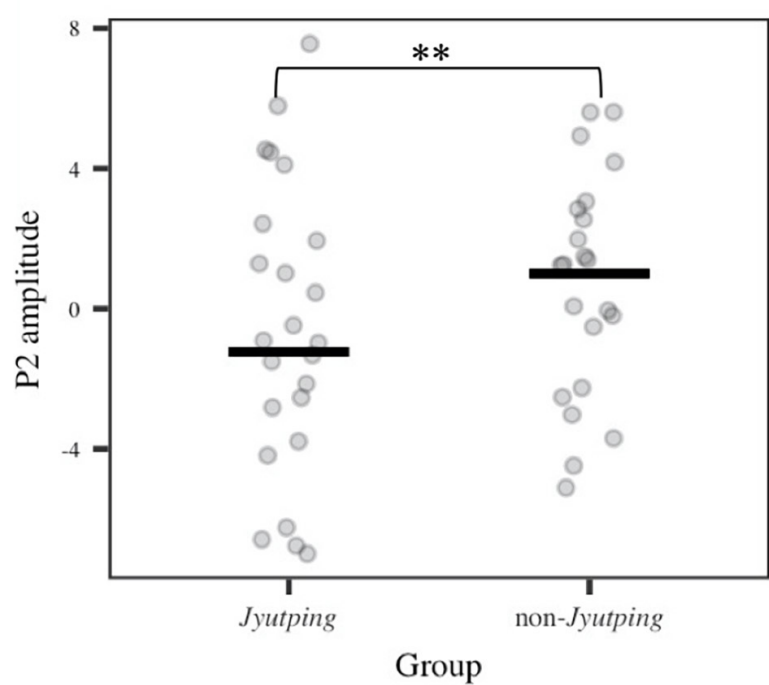

B

The group effect on $\mathrm{N} 1$

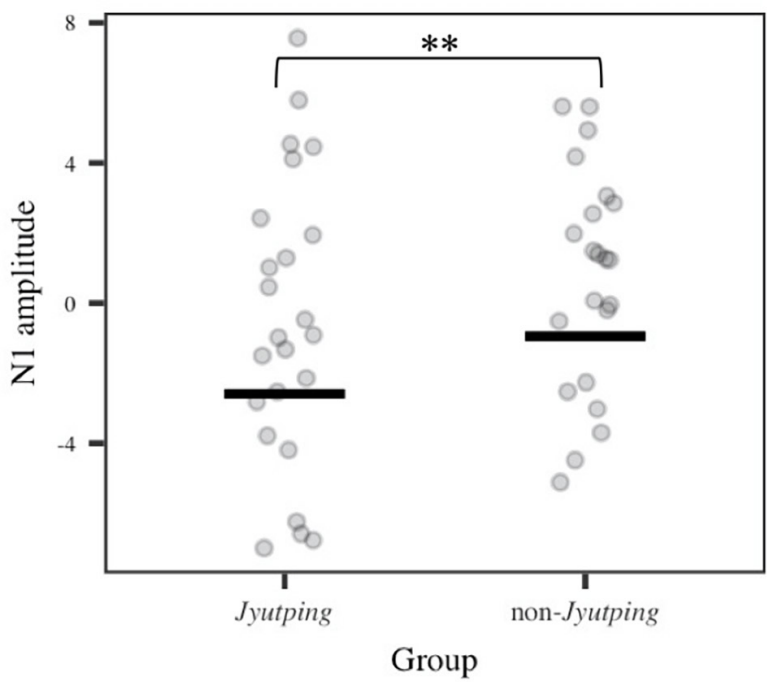

D The list effect on P2

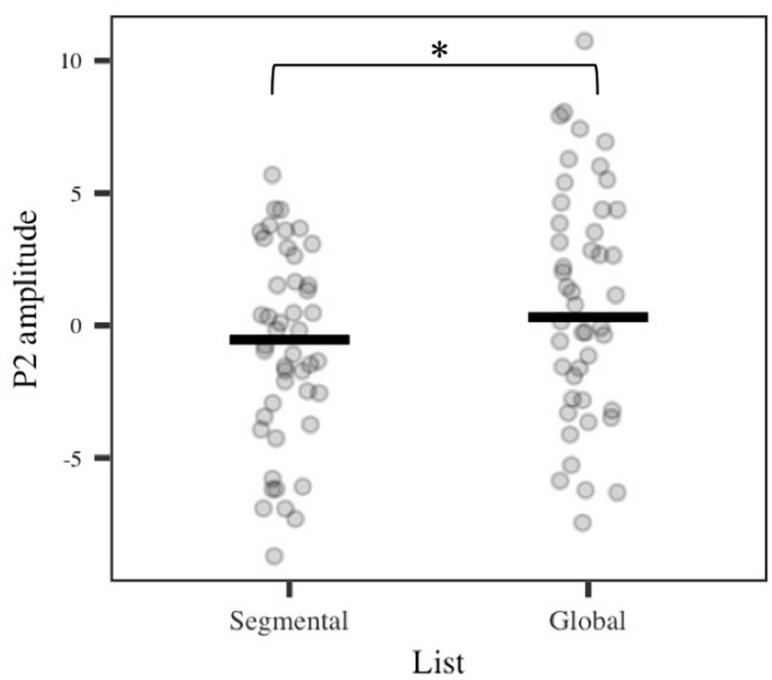

FIGURE 3 | Averaged ERP amplitude in the P1 (0-93 ms), N1 (95-237 ms), and P2 (239-353 ms) time-windows obtained from the fronto-central electrode cluster (F3, Fz, F1, FC1, FC2, and Cz). (A) Group and onset congruency effects on P1. (B) The group effect on N1. (C) The group effect on P2. (D) The list effect on P2. The solid line denotes means estimated from the linear model while each dot represents individual ERP amplitude. Significance levels: ${ }^{*} p<0.05 ;{ }^{* *} p<0.01$; ${ }^{\star \star \star} p<0.001$.

phonemic level become more explicitly accessible after the acquisition of an alphabetic script (Morais et al., 1979; Ziegler and Goswami, 2005; Morais, 2021). The ERP findings provide further insight on the temporal dynamics of the previously reported enhanced phonetic/phonological processing in Chinese readers with alphabetic literacy (Read et al., 1986; de Gelder et al., 1993; Ho and Bryant, 1997; Cheung et al., 2001; Brennan et al., 2013).

The sustained stronger negativity observed in the Jyutping group that emerged from the P1 time-window $(0-93 \mathrm{~ms})$ and continued until the $\mathrm{N} 1$ (95-237 ms) and P2 time-window (239$353 \mathrm{~ms}$ ) could be attributed to their enhanced ability to encode acoustic-phonetic information or to process phonological units at a more abstract fine-grained phonemic level. The P1-N1-P2 complex has been shown to encode phonetic features and index early stages of phonetic/phonological processing (Obleser et al., 2003; Tremblay et al., 2003; Toscano et al., 2010). For instance, the N1/N1m component has been reported to encode acousticphonetic features such as voice onset time (Toscano et al., 2010) and consonant/vowel place features (Obleser et al., 2003). 


\section{A ERP waveforms}
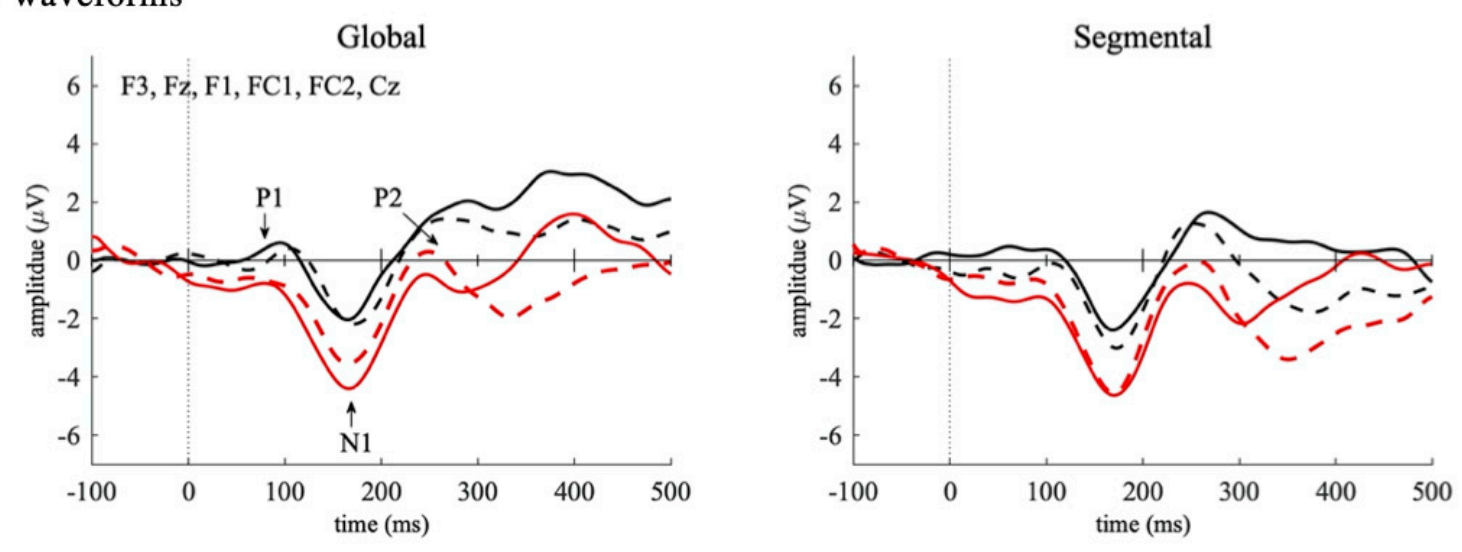

Jyutping group/congruent onsets
non-Jyutping group/congruent onsets
no

\section{B Topographic maps}

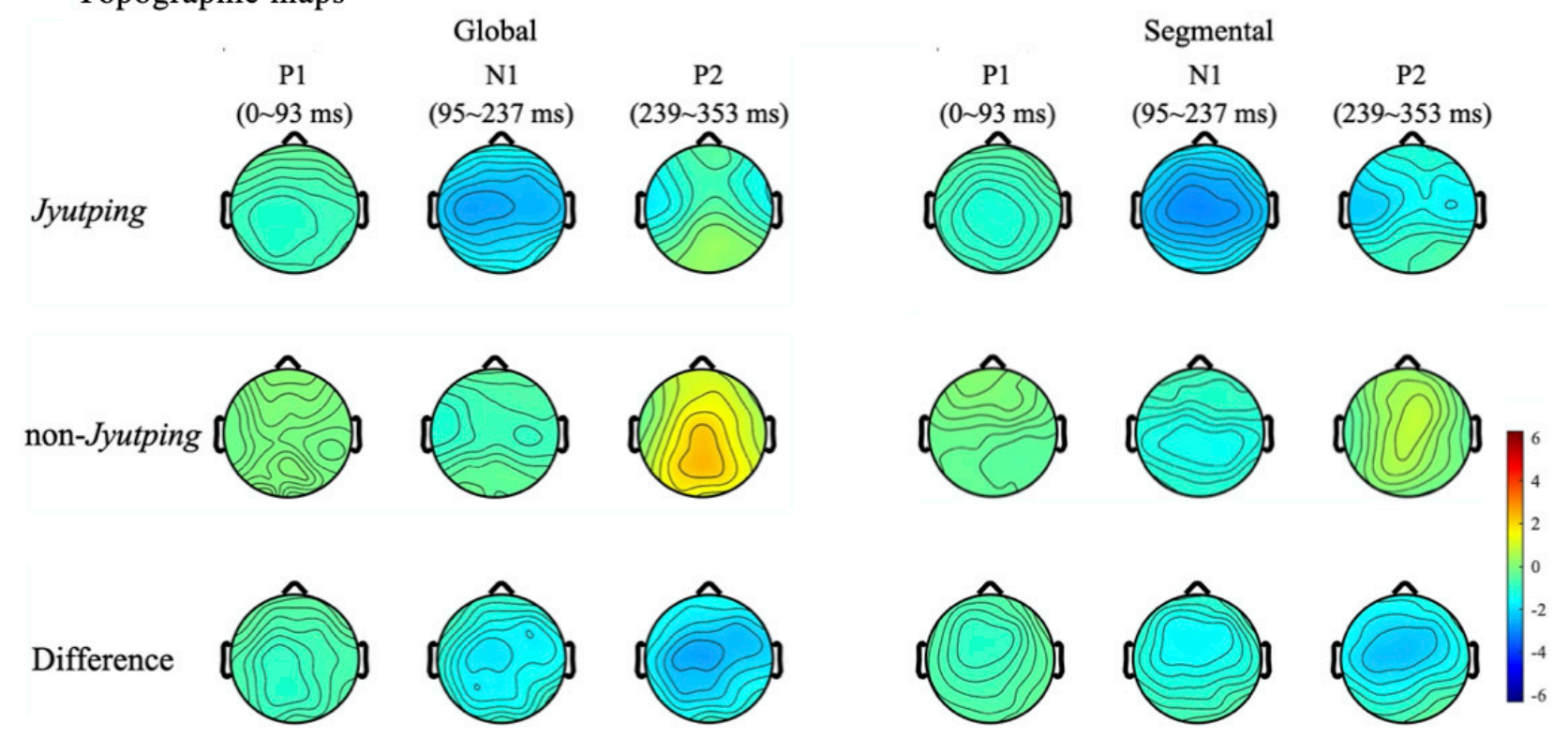

FIGURE 4 | Event-related potential waveforms and topographic maps illustrating effects in the P1-N1-P2 time windows. (A) ERP waveforms. The ERP waveforms are averaged from the fronto-central electrode cluster (F3, Fz, F1, FC1, FC2, and Cz). The three auditory ERPs-P1, N1, and P2-are marked in the waveforms shown in the left panel. (B) Topographic maps. The topographies are pooled across congruent and incongruent onsets. Each map shows the mean amplitude in the three time-windows-P1 (0-93 ms), N1 (95-237 ms), and P2 (239-353 ms).

Moreover, the neural generators of P1-N1-P2 lie in multiple auditory areas, like Heschl's gyrus, PT and STG, that are relevant for auditory analysis of speech (Martin et al., 2008). These neural sources are broadly consistent with the brain regions recruited during phonetic/phonological processing (e.g., PT and STG), which show augmented activations to speech in competent readers of alphabetic scripts compared with preliterate children, illiterate adults or readers of logographic scripts (Dehaene et al., 2010, 2015; Brennan et al., 2013; Monzalvo and DehaeneLambertz, 2013). Thus, the current ERP findings support the view that the acquisition of an alphabetic script can lead to enhanced acoustic-phonetic processing at a lower auditory level or the functional reorganization of the higher-level phonological network that is responsible for encoding more fine-grained phonological units like phonemes (Castro-Caldas et al., 1998; Petersson et al., 2000; Dehaene et al., 2010, 2015; Brennan et al., 2013; Monzalvo and Dehaene-Lambertz, 2013).

An alternative or complementary account is that the negativity observed in the time-windows of early auditory evoked potentials for the Jyutping group also reflects enhanced attention/awareness processes. Note that the Jyutping group exhibited more negative ERPs in the P2 time-window, instead of larger (or more positive) P2 as predicted. Enlarged P2 has been frequently reported to be associated with enhanced auditory or phonological processing, for example, in musicians or after laboratory training on speech or musical sounds 


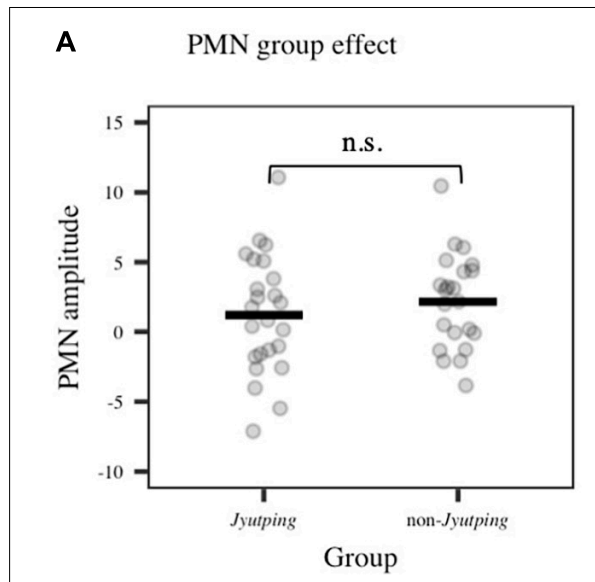

B PMN onset congruency effect

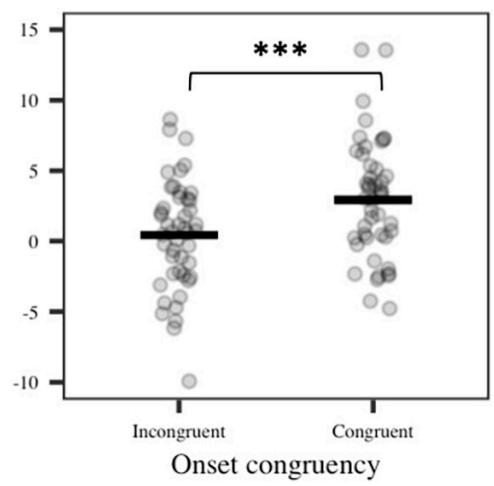

C PMN list effect

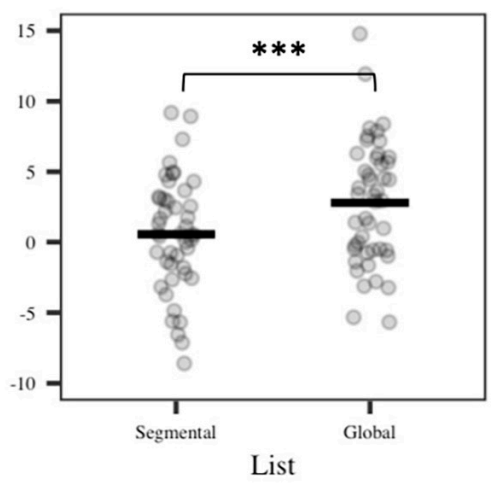

FIGURE 5 | Averaged ERP amplitude in the PMN time-window (355-500 ms) obtained from the centro-parietal electrode cluster (P3, Pz, P4, CP1, CP2, and Cz). (A) PMN group effect (non-significant, n.s.). (B) PMN onset congruency effect. (C) PMN list effect. The solid line denotes means estimated from the linear model while each dot represents individual ERP amplitude. Significance levels: ${ }^{\star} p<0.05 ;{ }^{\star \star} p<0.01 ;{ }^{\star \star \star} p<0.001$.

(Tremblay et al., 2001; Tremblay and Kraus, 2002; Shahin, 2011). One possible hypothesis is that the amplitude reduction in the P2 time-window for the Jyutping group may be related to a superimposed processing negativity (PN) wave, also known as negativity difference $(\mathrm{Nd})$, or contingent negative variation (CNV), that reflects top-down attentional processes (Tecce, 1972; Näätänen et al., 1978; Näätänen, 1982; Näätänen and Picton, 1987; Lange et al., 2003; Baumann et al., 2008). These relatively low-frequency waves can overlap early auditory ERPs. For example, Baumann et al. (2008) found a similar lowfrequency $\mathrm{PN}$-like or Nd-like component for musicians, which was superimposed on auditory P1-N1-P2 in their pitch, sine wave and instrumental tone discrimination tasks. As argued by the authors, the enlarged N1-P2 per se in musicians probably reflects an increase of neural resources for auditory feature encoding. Yet, this enhancement may be distinct from a superimposed $\mathrm{PN}$-like negative wave which reflects the plasticity of attentional control. They reasoned that if musical training leads to both enhanced positivity in P2 and negativity in a PNlike component, the different polarities of these two components could possibly explain their null effect of musical expertise in the P2 time-window. Consistent with the PN explanation, the overall group difference in our ERP data seems to manifest as a fronto-centrally distributed, prolonged negativity, which begins approximately at the stimulus onset and continues into later sensory/perceptual processing stages as indexed by auditory evoked potentials. Therefore, the attentional account captures the amplitude reduction in the P2 time-window or more generally the globally sustained negativity for the Jyutping group across the P1-N1-P2 time windows. Furthermore, the attentional account holds some support from neuroimaging studies demonstrating the engagement of the attentional network in meta-phonological tasks like auditory phoneme judgment (Burton et al., 2000). Literacy and phonological awareness skills have also been reported to modulate functional activations of the attentional network in a pseudoword repetition task which requires some meta-phonological skills (Castro-Caldas et al., 1998;
Petersson et al., 2000). These tasks that tap meta-phonological skills might require participants' abilities to shift their attention to phonology in general or more specifically to fine-grained phonological units like phonemes.

Besides the group effect, the behavioral data showed that the segmental list elicited more errors and slower responses than the global list, but these effects were only present for the trials with congruent onsets. These results suggested higher task requirements or greater difficulties for the segmental list (Burton et al., 2000). However, the segmental list is not uniformly more difficult than the global list. The trials in the congruent onset/segmental list condition are the most difficult probably due to the necessity of ignoring or inhibiting the relatively salient rhyme "difference" and focusing on the target onset dimension to arrive at a correct "same" onset response.

For ERP effects regarding list and onset congruency, the segmental list elicited more negative ERPs than the global list in the P2 time-window, probably reflecting higher demand on phonetic/phonological processing or attentional modulation in the segmental list. Another possibility is that the list effect in the P2 time-window was due to the partial overlap with the ensuing PMN component that is sensitive to the rhyme mismatch. The PMN component (355-500 ms) was modulated by both list and onset congruency differences, which can be explained by the rhyme and onset mismatch in the speech stimuli, respectively. A widely accepted theory is that the PMN represents phonological analysis of the acoustic features of speech sounds, which is affected by phonemic expectations established by prior contexts (Connolly and Phillips, 1994; Newman et al., 2003; Lafontaine et al., 2012; Zhang, 2018). Our finding that both onset and rhyme mismatches modulate the PMN amplitude is in line with this theory. Additionally, the PMN seems to index overall phonological mismatch in the speech signals regardless of its task relevance, i.e., the target onset dimension and the task-irrelevant rhyme dimension. Consistent with this idea, the PMN reported here is the strongest in the incongruent onset/segmental list condition, i.e., both onset and 


\section{A ERP waveforms}
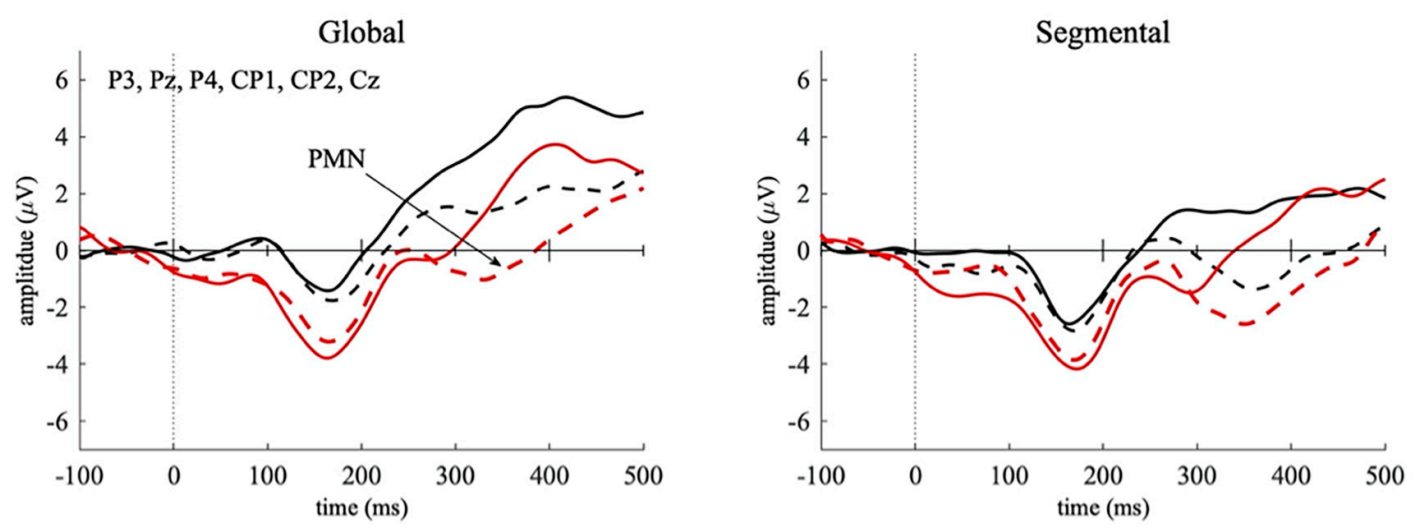

Jyutping group/congruent onsets
non-Jyutping group/congruent onsets
-

\section{B Topographic maps}
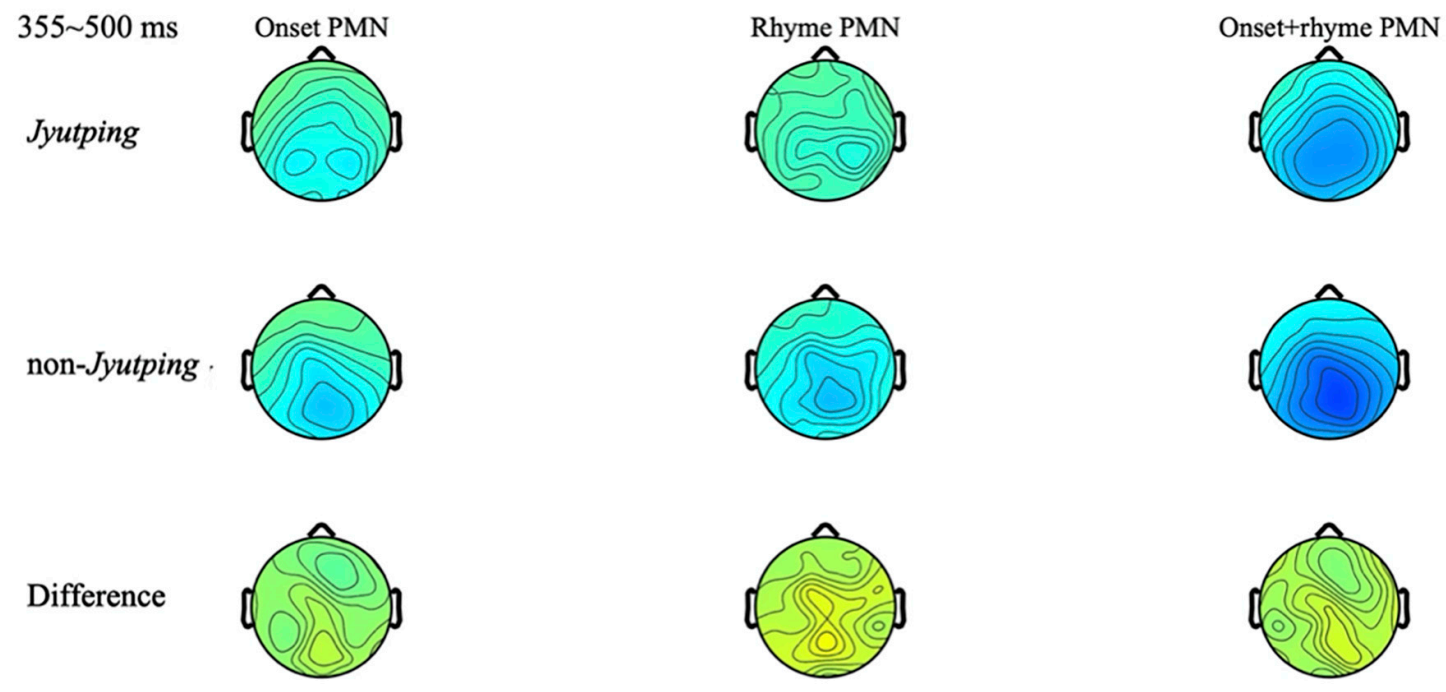

FIGURE 6 | Event-related potential waveforms and topographic maps illustrating the effects in the PMN time window. (A) ERP waveforms. The ERP waveforms are averaged from the centro-parietal electrode cluster (P3, Pz, P4, CP1, CP2, and Cz). The PMN component is marked in the waveforms shown in the left panel. (B) Topographic maps. The topographies illustrate the mean amplitude of the isolated PMN component in the time-window from 355 to 500 ms. Three isolated PMNs are displayed to better delineate the PMN effect-onset mismatch (incongruent onset/global list minus congruent onset/global list), rhyme mismatch (congruent onset/segmental list minus congruent onset/global list) and rhyme+onset mismatch (incongruent onset/segmental list minus congruent onset/global list). Note the congruent onset/global list condition, which has completely identical paired pseudowords without any phonological mismatch, is the baseline to derive the PMN component.

rhyme mismatches, although the behavioral results suggest that the congruent onset/segmental list condition should be the most difficult condition as discussed above.

Then, what could possibly explain the absence of a group difference for the PMN component? One explanation is that the locus of the influence of alphabetic literacy skills on phonemic processing is at an earlier acoustic-phonetic encoding stage as indexed by the P1-N1-P2, but not at a later phonological processing stage as indexed by the PMN. Another possibility is that the PMN per se is probably not specific to more abstract "phonemic" operations, like translating acoustics into phonemic categories, or detecting mismatch between phonemic expectations and incoming speech signals, as postulated by the current PMN theory (Connolly and Phillips, 1994; Newman et al., 2003; Lafontaine et al., 2012; Zhang, 2018). Instead, a mismatch between auditory traces in the working memory and the incoming speech signal might be sufficient to generate the PMN, regardless of the grain size of phonological representations.

We also predicted that there might be interactions among group, list and onset congruency in both the behavioral and ERP responses. Only the interaction between group and onset congruency in the P1 time window was consistent with this prediction. One possible explanation for this interaction effect 
is that congruent trials, especially those in the congruent onset/segmental list condition, might be more difficult and thus increase the attentional load or phonetic/phonological encoding demand at an early stage. However, further studies are needed to confirm this initial observation on the interaction between task difficulties or processing demands and the impact of alphabetic literacy on phonemic processing.

One potential limitation of the present cross-sectional study is that participants' Jyutping skills might be correlated with or affected by other pre-existing skills, like their knowledge of the English alphabet. For example, the two groups of participants were matched on their general knowledge of English that was assessed by standardized English tests. Nevertheless, we could not completely rule out the possibility that English phonological awareness and alphabetic skills might be correlated with their Cantonese phonological awareness and Jyutping knowledge, and further contribute to the behavioral and neural differences observed between the two groups. Indeed, many aspects of the cross-language transfer effects of phonological awareness and literacy skills remain controversial (including the direction of such transfer effects: L1-to-L2 or L2-to-L1), and the discrepancies could be due to multiple factors (e.g., the phonological and orthographic similarity between two languages; Bialystok et al., 2005; Dodd et al., 2008; Deng et al., 2019). The current finding confirmed the behavioral advantage of alphabetic literacy skills in speech processing in one's native language and provided further evidence on the underlying neuro-cognitive processes of such effects. Nevertheless, future research can employ a longitudinal design with an alphabetic literacy training paradigm and test participants' alphabetic/phonological skills in different languages before and after training. This design would help to segregate the influences of L1 and L2 literacy knowledge and phonological awareness skills on L1 speech processing and explore their possible interactions during learning. Furthermore, with this design researchers can further demonstrate the causal link between alphabetic literacy skills and phonological awareness enhancement, and to assess both their language-specific and language-independent components.

\section{CONCLUSION}

The major goal of the present study is to examine whether and how Chinese speakers' phonological awareness and the temporal unfolding of its underlying neuro-cognitive processes are sensitive to alphabetic literacy. We showed that logographic Chinese readers with Jyutping expertise performed auditory onset phoneme judgment more accurately. For ERP responses,

\section{REFERENCES}

Bates, D., Maechler, M., Bolker, B., and Walker, S. (2020). The lme4 Package. $R$ package version 1.1-25.

Baumann, S., Meyer, M., and Jäncke, L. (2008). Enhancement of auditory-evoked potentials in musicians reflects an influence of expertise but not selective attention. J. Cogn. Neurosci. 20, 2238-2249. doi: 10.1162/jocn.2008.20157 the Jyutping group exhibited more negative-going ERPs in early auditory P1-N1-P2 time-windows than the non-Jyutping group. The PMN component exhibited sensitivity to both onset and rhyme mismatches in the speech signals, but it was not modulated by alphabetic literacy skills. The early enhancement in the processing stream probably reflects the increased efficiency of phonetic/phonological encoding and the associated attention/awareness modulation.

\section{DATA AVAILABILITY STATEMENT}

The datasets presented in this study can be found in online repositories: https://osf.io/2pm7y/.

\section{ETHICS STATEMENT}

The studies involving human participants were reviewed and approved by The Human Subjects Ethics Sub-committee of the Hong Kong Polytechnic University. The participants provided their written informed consent to participate in this study.

\section{AUTHOR CONTRIBUTIONS}

YZ: formal analysis, visualization, and writing-original draft. CP: conceptualization, methodology, and writing-review and editing. MB and DL: conceptualization. K-YL and L-YY: methodology and investigation. CZ: conceptualization, writing-review and editing, supervision, and funding acquisition. All authors contributed to the article and approved the submitted version.

\section{FUNDING}

This work was supported by grants from the Research Grants Council of Hong Kong (ECS: 25603916), the Departmental General Research Funds-International Collaboration (P0008738) and the Departmental Reward Scheme for Research Publications in Indexed Journals, and was carried out under the aegis of the Labex BLRI (ANR-11-LABX-0036), the Institut Convergence ILCB (ANR-16-CONV-0002), and the Excellence Initiative of Aix-Marseille University (A*MIDEX).

\section{ACKNOWLEDGMENTS}

We thank Assal Habibi for constructive comments.

Bialystok, E., Luk, G., and Kwan, E. (2005). Bilingualism, biliteracy, and learning to read: interactions among languages and writing systems. Sci. Stud. Reading 9, 43-61. doi: 10.1207/s1532799xssr0901_4

Brennan, C., Cao, F., Pedroarena-Leal, N., Mcnorgan, C., and Booth, J. R. (2013). Reading acquisition reorganizes the phonological awareness network only in alphabetic writing systems. Hum. Brain Mapp. 34, 3354-3368. doi: 10.1002/ hbm. 22147 
Burton, M. W., Small, S. L., and Blumstein, S. E. (2000). The role of segmentation in phonological processing: an fMRI investigation. J. Cogn. Neurosci. 12, 679-690. doi: $10.1162 / 089892900562309$

Castro-Caldas, A., Petersson, K. M., Reis, A., Stone-Elander, S., and Ingvar, M. (1998). The illiterate brain. learning to read and write during childhood influences the functional organization of the adult brain. Brain 121, 1053-1063. doi: $10.1093 /$ brain/121.6.1053

Chen, J. Y., Chen, T. M., and Dell, G. S. (2002). Word-form encoding in Mandarin Chinese as assessed by the implicit priming task. J. Memory Lang. 46, 751-781. doi: 10.1006/jmla.2001.2825

Cheung, H., Chen, H. C., Lai, C. Y., Wong, O. C., and Hills, M. (2001). The development of phonological awareness: effects of spoken language experience and orthography. Cognition 81, 227-241. doi: 10.1016/S0010-0277(01)00136-6

Connolly, J. F., and Phillips, N. A. (1994). Event-related potential components reflect phonological and semantic processing of the terminal word of spoken sentences. J. Cogn. Neurosci. 6, 256-266. doi: 10.1162/jocn.1994.6.3.256

de Gelder, B., Vroomen, J., and Bertelson, P. (1993). The effects of alphabeticreading competence on language representation in bilingual Chinese subjects. Psychol. Res. 55, 315-321. doi: 10.1007/BF00419691

Dehaene, S., Cohen, L., Morais, J., and Kolinsky, R. (2015). Illiterate to literate: Behavioural and cerebral changes induced by reading acquisition. Nat. Rev. Neurosci. 16, 234-244. doi: 10.1038/nrn3924

Dehaene, S., Pegado, F., Braga, L. W., Ventura, P., Filho, G. N., Jobert, A., et al. (2010). How learning to read changes the cortical for vision and language. Science 330, 1359-1364. doi: 10.1126/science.1194140

Delorme, A., and Makeig, S. (2004). EEGLAB: an open source toolbox for analysis of single-trial EEG dynamics. J. Neurosci. Methods 13, 9-21. doi: 10.1016/j. jneumeth.2003.10.009

Deng, Q., Choi, W., and Tong, X. (2019). Bidirectional cross-linguistic association of phonological skills and reading comprehension: evidence from hong kong Chinese-English bilingual readers. J. Learn. Disabil. 52, 299-311. doi: 10.1177/ 0022219419842914

Dodd, B., So, L. K. H., and Lam, K. K. C. (2008). Bilingualism and learning: the effect of language pair on phonological awareness abilities. Australian J. Learn. Diff. 13, 99-113. doi: 10.1080/19404150802380514

Ho, C. S., and Bryant, P. (1997). Development of phonological awareness of Chinese children in Hong Kong. J. Psychol. Res. 26, 109-126. doi: 10.1023/a: 1025016322316

Lafontaine, H., Chetail, F., Colin, C., Kolinsky, R., and Pattamadilok, C. (2012). Role and activation time course of phonological and orthographic information during phoneme judgments. Neuropsychologia 50, 2897-2906. doi: 10.1016/j. neuropsychologia.2012.08.020

Lange, K., Rösler, F., and Röder, B. (2003). Early processing stages are modulated when auditory stimuli are presented at an attended moment in time: an eventrelated potential study. Psychophysiology 40, 806-817. doi: 10.1111/1469-8986. 00081

Lenth, R., Singmann, H., Love, J., Buerkner, P., and Herve, M. (2009). Emmeans: Estimated marginal means. R package version 1.4.2. doi: 10.1080/00031305.1980. 10483031

Liberman, I. Y., Shankweiler, D., Fischer, F. W., and Carter, B. (1974). Explicit syllable and phoneme segmentation in the young child. J. Exp. Child Psychol. 18, 201-212. doi: 10.1016/0022-0965(74)90101-5

Martin, B. A., Tremblay, K. L., and Korczak, P. (2008). Speech evoked potentials: From the laboratory to the clinic. Ear Hearing 29, 285-313. doi: 10.1097/AUD. 0b013e3181662c0e

McPherson, W. B., Ackerman, P. T., Holcomb, P. J., and Dykman, R. A. (1998). Event-related brain potentials elicited during phonological processing differentiate subgroups of reading disabled adolescents. Brain Lang. 62, 163185. doi: $10.1006 /$ brln.1997.1893

Meyer, A. S. (1991). The time course of phonological encoding in language production: phonological encoding inside a syllable. J. Memory Lang. 30, 69-89. doi: 10.1016/0749-596X(91)90011-8

Monzalvo, K., and Dehaene-Lambertz, G. (2013). How reading acquisition changes children's spoken language network. Brain Lang. 127, 356-365. doi: 10.1016/j. bandl.2013.10.009

Morais, J. (2021). The phoneme: a conceptual heritage from alphabetic literacy. Cognition doi: 10.1016/j.cognition.2021.104740
Morais, J., Cary, L., Alegria, J., and Bertelson, P. (1979). Does awareness of speech as a sequence of phones arise spontaneously? Cognition 7, 323-331. doi: 10. 1016/0010-0277(79)90020-9

Näätänen, R. (1982). Processing negativity: an evoked-potential reflection of selective attention. Psychol. Bull. 92, 605-640.

Näätänen, R., Gaillard, A. W., and Mäntysalo, S. (1978). Early selective-attention effect on evoked potential reinterpreted. Acta Psychol. 42, 313-329. doi: 10. 1109/CICN.2011.111

Nätänen, R., and Picton, T. W. (1987). The N1 wave of the human electric and magnetic response to sound: a review and an analysis of the component structure. Psychophysiology 24, 375-425. doi: 10.1111/j.1469-8986. 1987.tb00311.x

Newman, R. L., Connolly, J. F., Service, E., and McIvor, K. (2003). Influence of phonological expectations during a phoneme deletion task: evidence from event-related brain potentials. Psychophysiology 40, 640-647. doi: 10.1111/ 1469-8986.00065

Obleser, J., Lahiri, A., and Eulitz, C. (2003). Auditory-evoked magnetic field codes place of articulation in timing and topography around 100 milliseconds post syllable onset. NeuroImage 20, 1839-1847. doi: 10.1016/j.neuroimage.2003.07. 019

Onton, J., Westerfield, M., Townsend, J., and Makeig, S. (2006). Imaging human EEG dynamics using independent component analysis. Neurosci. Biobehav. Rev. 30, 808-822. doi: 10.1016/j.neubiorev.2006.06.007

Pattamadilok, C., Perre, L., and Ziegler, J. C. (2011). Beyond rhyme or reason: ERPs reveal task-specific activation of orthography on spoken language. Brain Lang. 116, 116-124. doi: 10.1016/j.bandl.2010.12.002

Peng, G., Zhang, C., Zheng, H.-Y., Minett, J. W., and Wang, W. S.-Y. (2012). The effect of inter-talker variations on acoustic-perceptual mapping in Cantonese and Mandarin tone systems. J. Speech Lang. Hear. Res. 55, 579-595. doi: 10. 1044/1092-4388(2011/11-0025)

Perfetti, C. A., and Bell, L. (1991). Phonemic activation during the first $40 \mathrm{~ms}$ of word identification: evidence from backward masking and priming. J. Memory Lang. 30, 473-485. doi: 10.1016/0749-596X(91)90017-E

Perfetti, C. A., Nelson, J., Liu, Y., Fiez, J., and Tan, L. (2010). "The neural bases of reading-universals and writing system variations," in The Neural Basis of Reading, 2nd Edn, eds C. Piers, H. Pete, K. Morten, and P. Ken (Oxford University Press), 147-172. doi: 10.1093/acprof:oso/9780195300369.003. 0006

Perfetti, C. A., and Tan, L. H. (1998). The time course of graphic, phonological, and semantic activation in Chinese character identification. J. Exp. Psychol. Learn. Memory Cogn. 24, 101-118. doi: 10.1037/0278-7393.24.1.101

Perre, L., Pattamadilok, C., Montant, M., and Ziegler, J. C. (2009). Orthographic effects in spoken language: On-line activation or phonological restructuring? Brain Res. 1275, 73-80. doi: 10.1016/j.brainres.2009.04.018

Petersson, K. M., Reis, A., Ėf, S. A., De, H., Maria, S., and Ingvar, M. (2000). Language processing modulated by literacy: a network analysis of verbal repetition in literate and illiterate subjects. Massachusetts Inst. Technol. J. Cogn. Neurosci. 12, 364-382. doi: 10.1162/089892900562147

Read, C., Zhang, Y.-F., Nie, H.-Y., and Ding, B.-Q. (1986). The ability to manipulate speech sounds depends on knowing alphabetic writing. Cognition 24, 31-44. doi: 10.1016/0010-0277(86)90003-X

Reis, A., and Castro-Caldas, A. (1997). Illiteracy: a cause for biased cognitive development. J. Int. Neuropsychol. Soc. JINS 3, 444-450. doi: 10.1017/ S135561779700444X

Shahin, A. (2011). Neurophysiological influence of musical training on speech perception. Front. Psychol. 2:126. doi: 10.3389/fpsyg.2011.00126

Shu, H., Peng, H., and McBride-Chang, C. (2008). Phonological awareness in young Chinese children. Dev. Sci. 11, 171-181. doi: 10.1111/j.1467-7687.2007. 00654.x

Singmann, H., Bolker, B., Westfall, J., Aust, F., and Ben-Shachar, M. S. (2020). afex: Analysis of Factorial Experiments. R Package Version 0.28-0.

Tecce, J. J. (1972). Contingent negative variation (CNV) and psychological processes in man. Psychol. Bull. 77, 73-108. doi: 10.1037/h0032177

Toscano, J. C., McMurray, B., Dennhardt, J., and Luck, S. J. (2010). Continuous perception and graded categorization: electrophysiological evidence for a linear relationship between the acoustic signal and perceptual encoding of speech. Psychol. Sci. 21, 1532-1540. doi: 10.1177/0956797610384142 
Tremblay, K. L., Friesen, L., Martin, B. A., and Wright, R. (2003). Test-retest reliability of cortical evoked potentials using naturally produced speech sounds. Ear Hearing 24, 225-232. doi: 10.1097/01.AUD.0000069229.84883.03

Tremblay, K. L., and Kraus, N. (2002). Auditory training induces asymmetrical changes in cortical neural activity. J. Speech Lang. Hearing Res. 45, 564-572. doi: 10.1044/1092-4388(2002/045)

Tremblay, K. L., Kraus, N., McGee, T., Ponton, C., and Otis, B. (2001). Central auditory plasticity: changes in the N1-P2 complex after speechsound training. Ear Hearing 22, 79-90. doi: 10.1097/00003446-20010400000001

Zhang, C. (2018). Online adjustment of phonetic expectation of lexical tones to accommodate speaker variation: a combined behavioural and ERP study. Lang. Cogn. Neurosci. 33, 175-195. doi: 10.1080/23273798.2017.1376752

Ziegler, J. C., and Goswami, U. (2005). Reading acquisition, developmental dyslexia, and skilled reading across languages: a psycholinguistic grain size theory. Psychol. Bull. 131, 3-29. doi: 10.1037/0033-2909. 131.1 .3
Conflict of Interest: The authors declare that the research was conducted in the absence of any commercial or financial relationships that could be construed as a potential conflict of interest.

Publisher's Note: All claims expressed in this article are solely those of the authors and do not necessarily represent those of their affiliated organizations, or those of the publisher, the editors and the reviewers. Any product that may be evaluated in this article, or claim that may be made by its manufacturer, is not guaranteed or endorsed by the publisher.

Copyright (c) 2021 Zhang, Pattamadilok, Lau, Bakhtiar, Yim, Leung and Zhang. This is an open-access article distributed under the terms of the Creative Commons Attribution License (CC BY). The use, distribution or reproduction in other forums is permitted, provided the original author(s) and the copyright owner(s) are credited and that the original publication in this journal is cited, in accordance with accepted academic practice. No use, distribution or reproduction is permitted which does not comply with these terms. 